\title{
Outcomes of Total Laparoscopic Hysterectomy: A Single-Surgeon Experience of Initial 50 Cases
}

\author{
Sana Ashfaq ${ }^{1}$, Mubashra Samina ${ }^{2}$, Maria Jabeen ${ }^{3}$, Shaheen Zafar $^{2}$ \\ 1. Obstetrics and Gynaecology, Atia General Hospital/Koohi Goth Hospital, Research and Training Center, Karachi, PAK \\ 2. Obstetrics and Gynaecology, Atia General Hospital, Karachi, PAK 3. Obstetrics and Gynaecology, Liaqat National \\ Hospital, Karachi, PAK
}

Corresponding author: Sana Ashfaq, sanaashfaq.0096@gmail.com

\section{Abstract}

\section{Introduction}

In this study, we reported a single surgeon experience of total laparoscopic hysterectomy (TLH) in terms of intraoperative and early postoperative outcomes and complications. In addition, we compared our results with published literature for a reevaluation of complications and outcomes.

\section{Material and methods}

This present prospective study was conducted on 50 patients who underwent TLH due to benign causes. Patients diagnosed with abnormal uterine bleeding (AUB), uterine fibroids, and post-menopausal bleeding (PMB) were included in this analysis. Patients were discharged after 24 hours of surgery if there were considered fit for discharge. The patients' age, co-morbidities, size of the uterus, additional procedure along with TLH, and postoperative complications were collected and analyzed. The follow-up period was three months, done on the tenth day after surgery, the thirtieth day, and then at three months.

\section{Results}

The mean age of our patients was $46.42 \pm 5.01$ years. The major indication of hysterectomy was fibroids diagnosed in 27 (54.0\%) patients and AUB in 18 (36.0\%) patients. Out of 50, $10(20.0 \%)$ patients had a previous cesarean section, and 4 (8.0\%) had a bilateral tubal ligation (BTL). Mean surgery duration was $124.26 \pm 44.74$ minutes. Mean hospital stay was $2.18 \pm 0.39$ days. Total complications occurred in five (10.0\%) patients, ureteric injury in one (2.0\%) patient, port-site infections in 2 (4.0\%), and vault infections in 2 (4.0\%) patients.

\section{Conclusion}

TLH is a safe procedure and can be performed with minimal complications in patients with benign uterine etiology.

Review began 12/29/2020 Review ended 01/02/2021 Published 01/12/2021

\section{(c) Copyright 2021}

Ashfaq et al. This is an open access article distributed under the terms of the Creative Commons Attribution License CC-BY 4.0., which permits unrestricted use, distribution, and reproduction in any medium, provided the original author and source are credited.
Categories: Obstetrics/Gynecology

Keywords: benign diseases, complications, total laparoscopic hysterectomy

\section{Introduction}

After a cesarean section, total laparoscopic hysterectomy (TLH) is the second most common procedure in gynecology departments [1]. Abnormal uterine bleeding (AUB), pelvic organ prolapse, leiomyoma, and cancer are routine indications for TLH [2]. TLH is the preferred approach over other procedures, such as laparotomy, because of fewer complications and higher access rates [3]. Vaginal hysterectomy is shown to have fewer complications than the laparoscopic approach but its access is limited in many procedures [4-5]. Vaginal hysterectomy is more difficult to perform in older women with prolapse, especially when the procedure involves adnexal surgery because pelvic access is difficult using the vaginal approach in these patients [6]. Therefore, TLH has now become the preferred approach for benign conditions.

However, despite these benefits and acceptable clinical outcomes, TLH has not gained widespread importance. Even in the USA, the number of TLHs procedures has decreased instead of increasing. In the USA, the number of TLHs performed was $15.5 \%$ in 2006, and it reduced to $8.6 \%$ in 2010 [2]. This may be due to the increase in robotic hysterectomy in the USA [2]. The other reasons are that the laparoscopic approach requires a steep learning curve and is challenging in obese patients [7]. Furthermore, there is also a risk of injury to the urinary tract during the procedure, and the operative time is prolonged as compared to other approaches [5]. Due to these reasons even in Europe, only a minor proportion of total procedures is done laparoscopically $[6,8]$.

With the passage of time, gynecology consultants have gained more experience, therefore, the operative 
time and complications of TLH have decreased. In the present study, we reported a single surgeon experience of TLH in terms of intraoperative and early postoperative outcomes and complications. In addition, we compared our results with published literature for the reevaluation of complications and outcomes after TLH.

\section{Materials And Methods}

This present prospective study was conducted on 50 patients who underwent TLH due to benign causes. Patients diagnosed with AUB, uterine fibroids, and postmenopausal bleeding (PMB) were included in this analysis while cases with malignancy were excluded. The study duration was from August 2018 to December 2019. Before surgery, all patients were informed about TLH and its potential benefits and written consent was taken for participation in the study.

Routine investigations, including complete clinical history, complete blood profile, ultrasonography, and biopsy studies, were done to rule out any malignant pathology.

A single consultant performed all laparoscopic procedures. All procedures were done by a consultant gynecologist. The procedure was done after inducing general anesthesia by placing the patient in the lithotomy position, with legs spread apart with small flexion at the hip joint (to obtain lateral movements during surgery). The following steps were followed during hysterectomy: coagulation of upper pedicles, opening of vesicoperitoneal folds, skeletonization of uterine vessels, coagulation of vessels, colpotomy, followed by specimen retrieval vaginally. For keeping the pneumoperitonimum, vaginal occlusion was done. The vaginal vault was closed by continuous suturing by laparoscopy.

After surgery, patients were discharged after 24 hours of surgery if they were considered as fit for discharge. The patients' age, co-morbidities, size of the uterus, additional procedure along with TLH, and postoperative complications were collected and analyzed. The follow-up period was three months, done on the tenth day after surgery, the thirtieth day, and then at three months.

For data analysis, we used the Statistical Package for the Social Sciences (SPSS) v23 (IBM Corp., Armonk NY). The analysis of variance (ANOVA) test was applied to compare mean operative time and hospital stay in patients with isolated TLH and those with concomitant procedures by considering $\mathrm{p}$-value $\leqslant 0.05$ as significant statistically.

\section{Results}

The mean age of patients was $46.42 \pm 5.01$ years. Hepatitis was the most common morbidity presented in nine (18\%) patients; hypertension and diabetes mellitus presented in five (10\%) and five (10\%) patients, respectively. The major indication of hysterectomy was fibroids diagnosed in 27 (54\%) patients and AUB in 18 (36\%). The full etiologic spectrum is given in Table 1. Regarding previous history, 10 (20\%) patients had a history of C-section, four (8\%) had a bilateral tubal ligation (BTL), one (2\%) had laparotomy, and one (2\%) had an appendectomy. Isolated TLH was done in 31 (62\%) patients, TLH+BSO in 17 (24\%) patients, $\mathrm{TLH}+\mathrm{RSO}$ in one (2\%) patient, and TLH+LSO in one (2\%) patients. The mean surgery duration was $124.26 \pm 44.74$ minutes (Table 1 ). The mean surgery time was not significantly different in TLH alone versus those with the concomitant procedure (Table 1 ). 


\section{Cureus}

Age

$46.42 \pm 5.01$

Comorbidities

Hepatitis B

$1(2.0 \%)$

Hepatitis C

$9(18.0 \%)$

Hypertension

$5(10.0 \%)$

Diabetes Mellitus

$5(10.0 \%)$

Indications of TLH

AUB

$18(36.0 \%)$

Uterine Fibroids

27 (54.0\%)

PMB

$01(2.0 \%)$

Adenomyosis

$03(6.0 \%)$

Cerebral Palsy (CP) Child

$01(2.0 \%)$

Size of Uterus

$13.16 \pm 2.39$

Previous Surgical History

C-Section

$10(20.0 \%)$

Bilateral Tubal Ligation (BTL)

$4(8.0 \%)$

Laparotomy

$1(2.0 \%)$

$1(2.0 \%)$

Laparoscopic Appendectomy

$31(62.0 \%)$

TLH

$17(24.0 \%)$

TLH+BSO

$01(2.0 \%)$

TLH+RSO

$01(2.0 \%)$

TLH+LSO

Duration of Surgery (mins)
$124.26 \pm 44.74$

\section{TABLE 1: Baseline study variables}

TLH, total laparoscopic hysterectomy; AUB, abnormal uterine bleeding; PMB, post-menopausal bleeding; BSO, bilateral salpingo-oophorectomy; $\mathrm{RSO}$, right salpingo-oophorectomy; LSO, left salpingo-oophorectomy

Total complications occurred in five (10\%) patients; there were no major complications and only minor complications were diagnosed. Out of five, a ureteric injury occurred in one (2\%) patient, port-site infections were diagnosed in two (4\%), and vault infections in two (4\%) patients. The mean hospital stay was $2.18 \pm 0.39$ days (Table 2). 


\section{Cureus}

\begin{tabular}{|l|l}
\hline Mean Hospital Stay (days) & $2.18 \pm 0.39$ \\
\hline Need of Laparotomy & 0 \\
Paralytic lleus & 0 \\
Bladder Injury & 0 \\
Ureteric Injury & $1(2.0 \%)$ (Rt. Ureter) \\
Visceral Injury & 0 \\
Mortality & 0 \\
Need for Re-operation & 0 \\
Port-Site Infections & $2(4.0 \%)$ \\
Vaginal Bleeding & 0 \\
Vault Infections & $2(4.0 \%)$ \\
Vaginal Evisceration & 0 \\
Re-suturing of Vault & 0 \\
Vault Hematoma & 0 \\
Port-Site Hernia & 0 \\
Blood Transfusion & 0 \\
\hline
\end{tabular}

\section{TABLE 2: Procedural complications of TLH}

TLH, total laparoscopic hysterectomy

The mean hospital stay was not influenced by the type of surgical procedure (Table 3).

\begin{tabular}{|c|c|c|c|c|c|}
\hline & TLH Alone & TLH+BSO & TLH+RSO & TLH+LSO & P-value \\
\hline Operative Time & $129.74 \pm 48.49$ & $111.58 \pm 33.94$ & 194.0 & 100 & 0.21 \\
\hline Hospital Stay & $2.22 \pm 0.42$ & $2.11 \pm 0.33$ & 2.0 & 2.0 & 0.74 \\
\hline
\end{tabular}

TABLE 3: Comparison of mean operative time and hospital stay in patients with TLH alone and with concomitant procedures.

TLH, total laparoscopic hysterectomy; BSO, bilateral salpingo-oophorectomy; RSO, right salpingo-oophorectomy; LSO, left salpingo-oophorectomy

\section{Discussion}

Laparoscopic procedures are routine practice in gynecology departments for nearly the last 30 years [8-9]. In some countries like Pakistan, the use of LH has increased over some decades. LH can be performed using several techniques. In 2003, Reich et al. defined the terminology of LH and defined TLH as a procedure in which the whole procedure is performed through a laparoscope except for the removal of the uterus [8].

In the present study, we reported a single surgeon's experience of intraoperative and postoperative outcomes of TLH in benign diseases. In the present study, the mean age was $46.42 \pm 5.01$ years. Mereu et al. reported a mean age of $49.6 \pm 6.5$ years among patients who underwent TLH due to benign diseases [10]. Shim et al. also reported a mean age of 48 years [11].

The most common indication of TLH in our study was uterine fibroids presented in 54\% cases and AUB in $36 \%$ cases. Agarwal et al. reported fibroids in $51.2 \%$ of patients, AUB in $28 \%$, adenomyosis in $19.2 \%$, and hyperplasia in $1.6 \%$ of patients who underwent TLH. While for TAH, they reported fibroids in $48 \%$, AUB in $30.4 \%$, adenomyosis in $20 \%$, and hyperplasia in $5.6 \%$ of patients [3]. 
In our study, the mean operative time was $124.26 \pm 44.74$ minutes. Studies have reported differences in mean operative time. Kim et al. reported a mean operative' time of $149.3 \pm 59$ minutes [12]. Ng et al. reported a mean time of 136 minutes [13]. Their operative time was longer than our study. While studies by Wallwiener et al. and Boosz et al. reported a shorter operative time $-103 \pm 36$ minutes and $104 \pm 44$ minutes, respectively [14-15].

Regarding complications, we did not encounter any incidence of conversion to laparotomy and hospital mortality in our case series. There were only three types of complications that we encountered: ureteric injury in $2 \%$, port-site infections in $4 \%$, and vault infections in $4 \%$ of patients with an overall complication rate of $10 \%$.

A study by Mereu et al. reported bladder injury in $0.3 \%$, and blood transfusion in $0.3 \%$ of patients, port-site hernia in $0.3 \%$, wound infections in $1.1 \%$, vaginal cuff dehiscence in $0.3 \%$, and vaginal cuff bleeding in $0.8 \%$ after TLH. The overall complication rate was $4.4 \%$. They did not report an incidence of conversion to laparotomy or any hospital mortality [10].

A study by Agarwal et al. reported that years of experience of TLH has a significant influence on the complication rate of TLH. They reported complications of $14 \%$ in the first year of TLH, and it reduced to only $4.3 \%$ after three years of experience. The rate of conversion to laparotomy in the first year was $9.6 \%$, and it decreased significantly within four years [3].

A study by Twijnstra et al. reported a need for laparotomy in $4.8 \%$ of patients, bladder injury in $1.4 \%$, ureteral injury in $0.4 \%$, and bowel injury in $0.5 \%$ of patients [16].

The mean hospital stay in our study was $2.18 \pm 0.39$ days. Our length of stay was shorter when compared to Kim et al. and Wallwiener et al., who reported a hospital stay of 5.5 \pm 2.0 days and $4.9 \pm 2.8$ days, respectively $[12,14]$. This was comparable to the studies by Mereu et al., who reported a hospital stay of $2.6 \pm 1.1$ days [10].

The major limitation of this study is its non-randomized nature and small sample size because of the low volume of patient flow for hysterectomies in our hospital. However, we have compared our results with international literature, which has enabled us to verify the outcomes of TLH in our patients.

\section{Conclusions}

TLH is a safe procedure and can be performed with a minimal number of complications. Initial studies reported a higher complication rate, but the latest studies, including the present one, have found a very low rate of procedural complications. So this procedure can be adopted as the preferred approach in patients planned for hysterectomy due to benign diseases.

\section{Additional Information}

\section{Disclosures}

Human subjects: Consent was obtained by all participants in this study. Ethical Review Committee, Koohi Goth Hospital, Research \& Training Centre, Karachi issued approval 2018-0091. Animal subjects: All authors have confirmed that this study did not involve animal subjects or tissue. Conflicts of interest: In compliance with the ICMJE uniform disclosure form, all authors declare the following: Payment/services info: All authors have declared that no financial support was received from any organization for the submitted work. Financial relationships: All authors have declared that they have no financial relationships at present or within the previous three years with any organizations that might have an interest in the submitted work. Other relationships: All authors have declared that there are no other relationships or activities that could appear to have influenced the submitted work.

\section{References}

1. Chattopadhyay S, Patra KK, Halder M, Mandal A, Pal P, Bhattacharyya S: A comparative study of total laparoscopic hysterectomy and nondescent vaginal hysterectomy for treatment of benign diseases of uterus. Int J Reprod Contracept Obstet Gynecol. 2017, 3:1109-1112.

2. Wright JD, Herzog TJ, Tsui J, et al.: Nationwide trends in the performance of inpatient hysterectomy in the United States. Obstet Gynecol. 2013, 122:233-241. 10.1097/AOG.0b013e318299a6cf

3. Agarwal P, Bindal N, Yadav R: Risks and benefits of total laparoscopic hysterectomy and the effect of learning curve on them. J Obstet Gynaecol India. 2016, 66:379-384. 10.1007/s13224-015-0706-9

4. Chen B, Ren D-P, Li J-X, Li C-D: Comparison of vaginal and abdominal hysterectomy: a prospective nonrandomized trial. Pak J Med Sci. 2014, 30:875-879. 10.12669/pjms.304.4436

5. Aarts JW, Nieboer TE, Johnson N, Tavender E, Garry R, Mol BW, Kluivers KB: Surgical approach to hysterectomy for benign gynaecological disease. Cochrane Database Syst Rev. 2015, 8:003677. 10.1002/14651858.CD003677.pub5

6. Hoffmann E, Bennich G, Larsen CR, Lindschou J, Jakobsen JC, Lassen PD: 3-dimensional versus conventional laparoscopy for benign hysterectomy: protocol for a randomized clinical trial. BMC Womens Health. 2017, 17:76. 10.1186/s12905-017-0434-7 
7. McDonnell RM, Hollingworth JL, Chivers P, Cohen PA, Salfinger SG: Advanced training of gynecologic surgeons and incidence of intraoperative complications after total laparoscopic hysterectomy: a retrospective study of more than 2000 cases at a single institution. J Minim Invasive Gynecol. 2018, 25:810815. 10.1016/j.jmig.2017.12.005

8. Reich H: Laparoscopic hysterectomy: the big cutoff in laparoscopic surgery development . Minimally Invasive Gynecology. Gomes-da-Silveira G, da Silveira G, Pessini S (ed): Springer, Cham, Switzerland; 2018. 5-15. 10.1007/978-3-319-72592-5_2

9. Anbreen F, Qadir S, Naeem H, Farhat N, Ghafoor M, Hassan S: Type, time-trend and indications of hysterectomy. Gomal J Med Sci. 2018, 16:92-96.

10. Mereu L, Carlin R, Pellegrini A, Guasina F, Berlanda V, Tateo S: Total laparoscopic hysterectomy for benign disease: outcomes and literature analysis. Gynecol Surg. 2018, 15:19. 10.1186/s10397-018-1052-6

11. Shim SH, Suh JH, Park JE, Lee SJ, Lee JY, Kim SN, Kang SB: Predictors of 30-day morbidity after hysterectomy for benign disease. Int J Gynecol Obstet. 2019, 144:302-308. 10.1002/ijgo.12752

12. Kim SM, Park EK, Jeung IC, Kim CJ, Lee YS: Abdominal, multi-port and single-port total laparoscopic hysterectomy: eleven-year trends comparison of surgical outcomes complications of 936 cases. Arch Gynecol Obstet. 2015, 291:1313-1319. 10.1007/s00404-014-3576-y

13. Ng CC, Chern BS, Siow AY: Retrospective study of the success rates and complications associated with total laparoscopic hysterectomy. J Obstet Gynaecol Res. 2007, 33:512-518. 10.1111/j.1447-0756.2007.00577.x

14. Wallwiener M, Taran F-A, Rothmund R, et al.: Laparoscopic supracervical hysterectomy (LSH) versus total laparoscopic hysterectomy (TLH): an implementation study in 1,952 patients with an analysis of risk factors for conversion to laparotomy and complications, and of procedure-specific re-operations. Arch Gynecol Obstet. 2013, 288:1329-1339. 10.1007/s00404-013-2921-x

15. Boosz A, Lermann J, Mehlhorn G, et al.: Comparison of re-operation rates and complication rates after total laparoscopic hysterectomy (TLH) and laparoscopy-assisted supracervical hysterectomy (LASH). Eur J Obstet Gynecol Reprod Biol. 2011, 158:269-273. 10.1016/j.ejogrb.2011.04.021

16. Twijnstra AR, Blikkendaal MD, van Zwet EW, van Kesteren PJ, de Kroon CD, Jansen FW: Predictors of successful surgical outcome in laparoscopic hysterectomy. Obstet Gynecol. 2012, 119:700-708. 10.1097/AOG.0b013e31824b1966 